26. - Sur le probleme de Cauchy pour l'equation de Poisson et l'equation des ondes, C. R. Acad. Sci. Paris vol. 234 (1952) pp. 2584-2585.

27. - On the wave equation and the equation of Euler-Poisson, Proceedings of the Fifth Symposium in Applied Mathematics, 1952 (in print).

28. E. T. Whittaker and G. N. Watson, Modern analysis, New York, 1948.

29. S. Zaremba, Rendiconti dei Accademia dei Lincei (5) vol. 14 (1915) p. 904.

UNIVERSITY OF MARYLAND

\title{
CONSTRUCTION OF SOLUTIONS AND PROPAGATION OF ERRORS IN NONLINEAR PROBLEMS
}

\author{
H. D. BLOCK
}

1. Introduction. The purpose of this paper is to provide theoretical tools which will be useful, in practical applications, for approximating the solutions of a certain class of nonlinear equations.

2. Definitions. Let $(U,+, d)$ denote a mathematical system consisting of a set $U$ on whose elements are defined (i) a binary operation, + , such that $U$ is an Abelian group with respect to this operation and (ii) a metric function $d$, with respect to which $U$ is a metric space. If $d$ is invariant under translation (i.e., for any $u_{1}, u_{2}, u_{3}$, in $U$, $\left.d\left(u_{1}, u_{2}\right)=d\left(u_{1}+u_{3}, u_{2}+u_{3}\right)\right)$, then we shall call $(U,+, d)$ a $b$-space. Under these conditions we shall, for brevity, say that $U$ is a b-space. The group identity will be denoted by $\theta$, and $d(u, \theta)$ will be denoted by $\|u\|$.

If $\mathfrak{M}$ and $\mathfrak{N}$ are sets, and $K$ is a single-valued function defined for each $\mathfrak{m}$ of $\mathfrak{M}$ and having its values (which are denoted by $K \mathfrak{m}$ or $K(\mathfrak{m}))$ in $\mathfrak{R}$ then we shall say that $K$ maps $\mathfrak{M}$ into $\mathfrak{N}$.

Let $U$ and $V$ be $b$-spaces and let $K$ map $U$ into $V$. We define $m(K)$ as the infimum, and $M(K)$ as the supremum, of the quantity $\left\|K u_{1}-K u_{2}\right\| /\left\|u_{1}-u_{2}\right\|$ taken over all $u_{1}, u_{2}$ in $U$ with $u_{1} \neq u_{2}$. If $M(K)<\infty$, then we shall say that $K$ is bounded. The space $V$ is complete if each Cauchy sequence in $V$ has a limit in $V$. If $K$ is bounded and if, for each bounded set $\cup_{\alpha} u_{\alpha}$ in $U$, the set of images, $\cup_{\alpha}\left(K u_{\alpha}\right)$, contains a sequence which converges to an element in $V$, then $K$ is said to be completely continuous. We shall say that $V$ is complete for $K$ if, for each Cauchy sequence $\left\{u_{n}\right\}$ in $U$, the sequence $\left\{K u_{n}\right\}$ has a limit in $V$.

Presented to the Society, December 29, 1952; received by the editors November 20, 1952. 
REMARK 1. It is easy to show that: (a) if $V$ is a complete space and $K$ is bounded, then $V$ is complete for $K$; (b) if $K$ is completely continuous, then $V$ is complete for $K$.

We shall say that $K$ is closed if the conditions $\lim \left\{u_{n}\right\}=u$ and $\lim \left\{K u_{n}\right\}=v$ together imply that $K u=v$. We denote by $I_{U}$ the function defined, for each $u$ in $U$, by $I_{U}(u)=u$. If $K_{1}$ and $K_{2}$ each map $U$ into $V$, then $K_{1}+K_{2}$ and $K_{1}-K_{2}$ are mappings, defined for each $u$ in $U$ by the equations $\left(K_{1}+K_{2}\right) u=K_{1} u+K_{2} u$ and $\left(K_{1}-K_{2}\right) u$ $=K_{1} u-K_{2} u$.

We assume throughout this paper that $X$ and $Y$ are fixed $b$-spaces (not necessarily distinct), that $F$ is a given mapping from $X$ into $Y$, and that $m(F)=f>0$.

3. Solution of equations. We consider the equation

$$
F x=y .
$$

Theorem 1. If there exists a b-space $Z$, a mapping $A$ from $Y$ into $Z$ and a mapping $B$ from $Z$ into $X$ such that $m(A)=a>0, M(R)=r<1$, where $R=I_{Z}-A F B$, and if either of the following two conditions are satisfied $\left(c_{1}\right) Z$ is complete for $R$ or $\left(c_{2}\right) X$ is complete for $B$, and $F$ is closed; then, for each $y$ in $Y$, (i) the sequence $\left\{x_{n}\right\}$ defined by $x_{n+1}$ $=B z_{n+1}$, where $z_{n+1}=R z_{n}+A y$ and $z_{0}$ is an arbitrary element of $Z$, converges, say to $x$, (ii) $x$ is a unique solution of equation (1), (iii) $\left\|x-x_{n}\right\| \leqq(r(1+r) / a f(1-r))\left\|z_{n}-z_{n-1}\right\| \leqq\left(r^{n}(1+r) / a f(1-r)\right)\left\|z_{1}-z_{0}\right\|$.

Proof. We first note that $M(B) \leqq(1+r) / a f$, since for any $u$ and $v$ in $Z, \quad$ af $\|B u-B v\| \leqq\|A F B u-A F B v\|=\|u-v+R v-R u\| \leqq(1+r)$ $\cdot\|u-v\|$. Next $\left\|z_{n+1}-z_{n}\right\|=\left\|R z_{n}-R z_{n-1}\right\| \leqq r\left\|z_{n}-z_{n-1}\right\| \leqq \cdots . r^{n} \| z_{1}$ $-z_{0} \|$. Hence $\left\|z_{n+k}-z_{n}\right\| \leqq \sum_{i=1}^{k}\left\|z_{n+i}-z_{n+i-1}\right\| \leqq\left(\sum_{i=1}^{k} r^{i}\right)\left\|z_{n}-z_{n-1}\right\|$ $\leqq(r /(1-r))\left\|z_{n}-z_{n-1}\right\| \leqq\left(r^{n} /(1-r)\right)\left\|z_{1}-z_{0}\right\|$. Also $\left\|x_{n+k}-x_{n}\right\|$ $=\left\|B z_{n+k}-B z_{n}\right\| \leqq(r(1+r) / a f(1-r))\left\|z_{n}-z_{n-1}\right\| \leqq\left(r^{n}(1+r) / a f(1-r)\right)$ - $\left\|z_{1}-z_{0}\right\|$. Hence, the sequences $\left\{z_{n}\right\}$ and $\left\{x_{n}\right\}$ each converge in the sense of Cauchy. Now we verify (i). If we assume the hypothesis $\left(c_{1}\right)$ then $\left\{R z_{n}\right\}$ converges. But $z_{n+1}=R z_{n}+A y$, so that $\left\{z_{n}\right\}$ converges, say to $z$. Let $B z=x$. Then $\left\|x-x_{n}\right\|=\left\|B z-B z_{n}\right\| \leqq M(B)\left\|z-z_{n}\right\|$ so that $\left\{x_{n}\right\}$ converges to $x$. Assuming $\left(c_{2}\right)$, we see that $\left\{B z_{n}\right\}$ converges, say to $x$. Since $x_{n}=B z_{n},\left\{x_{n}\right\}$ converges to $x$. We next verify (ii). There is at most one solution of equation (1) since $F x^{\prime}=F x$ implies $0=\left\|F x^{\prime}-F x\right\| \geqq f\left\|x^{\prime}-x\right\|$; i.e. $x^{\prime}=x$. Assuming $\left(c_{1}\right)$ we have seen that $\left\{z_{n}\right\}$ converges to $z$ and $\left\{x_{n}\right\}$ converges to $x=B z$. Hence for this case $a\|F x-y\| \leqq\|A F B z-A y\|=\left\|A F B z-z_{n+1}+R z_{n}\right\| \leqq\left\|z-z_{n+1}\right\|+r \| z_{n}$ $-z \|$, so that $F x=y$. Assuming $\left(c_{2}\right)$ we have $z_{n+1}-z_{n}=A y-A F B z_{n}$ so that $a\left\|y-F x_{n}\right\| \leqq\left\|z_{n+1}-z_{n}\right\|$. Hence $\left\{F x_{n}\right\}$ approaches $y$ while $\left\{x_{n}\right\}$ 
approaches $x$ so that $F x=y$. (iii) Let $k$ approach infinity in the estimate above for $\left\|x_{n+k}-x_{n}\right\|$.

REMARK 2. The conclusion (iii) of Theorem 1 may be interpreted as follows: If an error of $\epsilon$ can be tolerated in the calculation of $x$, then the iteration may be stopped when

$$
\left\|z_{n}-z_{n-1}\right\| \leqq a f(1-r) \epsilon / r(1+r) .
$$

One can also be sure, after having computed only $z_{1}$, that the number of iterations required will not exceed

$$
\left(\log [\epsilon a f(1-r)]-\log \left[(1+r)\left\|z_{1}-z_{0}\right\|\right]\right) / \log r .
$$

If $\bar{x}$ is chosen arbitrarily in $X$ as an approximation for $x$ (e.g. an intuitive modification of an $x_{n}$ ), an estimate of the error is provided by the inequality $\|x-\bar{x}\| \leqq(1 / f)\|y-F \bar{x}\|$. If we take $\bar{x}=\theta$ in the last inequality, then we have, in advance of the computation, an estimate of the magnitude of the solution sought.

REMARK 3. If the range of $F$ is $Y$, then $F$ has a right inverse mapping. Then one can choose this mapping as $B$, set $Z=Y$ and $A=I_{Y}$; the hypotheses of Theorem 1 (using $c_{1}$ ) will then be satisfied. Thus the quantities $Z, A, B$ mentioned in the theorem will exist if, and only if, the range of $F$ is $Y$.

REMARK 4. In virtue of Remark 1 , it follows that the alternatives $\left(c_{1}\right),\left(c_{2}\right)$ of the theorem can be replaced by the four alternatives: ( $\left.\mathrm{c}_{1}^{\prime}\right) Z$ is complete, $\left(\mathrm{c}_{1}^{\prime \prime}\right) R$ is completely continuous, $\left(\mathrm{c}_{2}^{\prime}\right) X$ is complete and $F$ is closed, $\left(c_{2}^{\prime \prime}\right) B$ is completely continuous and $F$ is closed.

This remark explains the common basis of the two settings in which linear problems are treated. ${ }^{1}$ Furthermore, it is not difficult to find a mapping such that $V$ is not a complete space, $K$ is not completely continuous, but $V$ is complete for $K$.

REMARK 5. We present here a simple illustration of an application of the theorem, reserving a more extended study of applications for a subsequent paper. Let $\Im$ denote the interval $0 \leqq t \leqq T$ and $\mathfrak{E}$ the interval $-\infty<\tau<\infty$. Let $C, D$, and $E$ each map $\mathfrak{S} \times \mathfrak{S}$ into $\mathfrak{W}$. Assume that each is continuous and that there are numbers $c, d, e$ such that, for each $t$ in $\Im$ and each $\tau$ and $\sigma$ in $\mathfrak{S},|C(\tau, t)-C(\sigma, t)| \leqq c|\tau-\sigma|$, $|D(\tau, t)-D(\sigma, t)| \leqq d|\tau-\sigma|,|E(\tau, t)-E(\sigma, t)| \leqq e|\tau-\sigma|$. Let $\mathfrak{S}^{n}$ denote the class of functions having a continuous $n$th derivative on $\Im$. Assume that $p \geqq 0$ and that $\alpha=c+d T+p T+e T^{2}<1$ and $\beta=2 c$ $+2 d T+e T^{2}<1$. Assume also that $y(t) \in \mathbb{E}^{\circ}$ (the 0 th derivative of a

1 Cf. A. T. Lonseth, The propagation of error in linear problems, Trans. Amer. Math. Soc. vol. 62 (1947) p. 194, lines 32-35. 
function is the function itself). Let $\dot{x}$ denote $d x / d t$ and $\ddot{x}$ denote $d^{2} x / d t^{2}$. Problem: Solve the equation

$$
\ddot{x}+C(\ddot{x}, t)+D(\dot{x}, t)+p \dot{x}+E(x, t)=y(t)
$$

for $x(t)$ in $\mathbb{S}^{2}$ with $x(0)=\dot{x}(0)=0$.

To apply the theorem, let $X$ be the subset of $\mathbb{S}^{2}$ having the property that for each $\xi(t)$ in $X, \xi(0)=\xi(0)=0$. Let $Y$ and $Z$ be $\mathfrak{S}^{0}$. In each case let $\|g\|=\max _{t \in \Im}|g(t)|$. For each $\xi$ in $X$ let $F \xi=\ddot{\xi}+C(\ddot{\xi}, t)$ $+D(\xi, t)+p \xi+E(\xi, t)$. One has for each $u$ and $v$ in $X$ and each $t$ in $\Im$, $|F u-F v| \geqq|\ddot{u}-\ddot{v}|-c\|\ddot{u}-\ddot{v}\|-(d+p)\|\dot{u}-\dot{v}\|-e\|u-v\|$, so that $\|F u-F v\| \geqq\left((1-\alpha) / T^{2}\right)\|u-v\|$. For each $\zeta$ in $\mathfrak{(}^{0}$ let

$$
B \zeta=\int_{0}^{t}(t-s) \zeta(s) d s \text { and } A \zeta=\zeta(t)-p \int_{0}^{t} e^{-p(t-s)} \zeta(s) d s .
$$

Then $m(A) \geqq e^{-p T}$, and $M(R) \leqq \beta\left(1-\left(e^{-p T} / 2\right)\right)$. Finally $Z$ is a complete space (cf. Remark 4), so that all the conditions of the theorem are satisfied.

4. Implicit functions. Let $\mathfrak{T}$ be a non-null set and let $G$ map $\mathfrak{I} \times X$ into $Y$. We now consider the equation

$$
G(t, \mathfrak{X})=\theta \text {. }
$$

If there is one and only one function $g$, mapping $\mathfrak{T}$ into $X$, such that, for each $t$ in $\mathfrak{T}, G(t, g(t))=\theta$, then we shall say that equation (2) defines the function $\mathrm{g}$.

For a fixed $t$ in $\mathfrak{T}$, the function $G_{t}$ defined, for each $u$ in $X$, by $G_{t} u$ $=G(t, u)$ maps $X$ into $Y$. The following theorem is equivalent to Theorem 1.

THEOREM 1'. Let $G$ be given. If, for each fixed $t$ in $\mathfrak{T}$, the hypotheses of Theorem 1 are satisfied (with $F$ there replaced by $G_{t}$ ) then equation (2) defines a function $g$. For each fixed $t$ in $\mathfrak{T}$ the computation of $\mathfrak{X}=g(t)$ may be carried out as in Theorem 1 , namely $\mathfrak{X}_{n+1}=B \zeta_{n+1}$, where $\zeta_{n+1}=\zeta_{n}$ $-A G\left(t, B \zeta_{n}\right)+A(\theta)$ and $\zeta_{0}$ is an arbitrary element of $Z$. Estimates of the error corresonding to conclusion (iii) of Theorem 1 are valid.

It is clear that Theorem 1 implies Theorem $1^{\prime}$. To verify the converse, let $\boldsymbol{y}$ be given. Let $\mathfrak{T}$ consist of the single element $\tau$ and define $G(\tau, x)=F x-y$. Then if $Z, A$, and $B$ exist satisfying the hypotheses of Theorem 1, define, for each $u$ in $Y, A^{\prime} u=A(u+y)$. Then $m\left(A^{\prime}\right)$ $=m(A)$ and $A^{\prime} G_{\tau}=A F$, so that the hypotheses of Theorem $1^{\prime}$ are satisfied with the available $Z$ and $B$ and with $A$ replaced by $A^{\prime}$. The conclusions of Theorem $1^{\prime}$ then imply the conclusions of Theorem 1. 
TheOREM 2. Let equation (2) define the function $g$ and let $\mathfrak{X}=g(t)$. Let $t_{0}$ be a fixed element of $\mathfrak{T}$ and $\mathfrak{X}_{0}=g\left(t_{0}\right)$. If $\mathfrak{T}$ is a space in which convergence is defined, and if whenever $t$ approaches $t_{0},\left\|G\left(t, \mathfrak{X}_{0}\right)\right\| / m\left(G_{t}\right)$ approaches zero, then $g$ is continuous at $t_{0}$; in particular if $\mathfrak{T}$ is a neighborhood space and if there is a neighborhood $N$ of $t_{0}$ such that inf $t_{t \in N} m\left(G_{t}\right)$ $>0$ and if the function $G_{\mathfrak{x}_{0}}$ from $\mathfrak{T}$ into $Y$ defined for each $t$ in $\mathfrak{T}$ by $G_{\mathfrak{X}_{0}}(t)=G\left(t, \mathfrak{X}_{0}\right)$ is continuous at $t_{0}$, then $g$ is continuous at $t_{0}$.

Proof.

$$
\begin{aligned}
\left\|\mathfrak{X}_{0}-\mathfrak{X}\right\| & \leqq \frac{1}{m\left(G_{t}\right)}\left\|G_{t_{1}} \mathfrak{X}_{0}-G_{t} g(t)\right\| \\
& =\frac{\left\|G\left(t, \mathfrak{X}_{0}\right)\right\|}{m\left(G_{t}\right)}=\frac{\left\|G\left(t, \mathfrak{X}_{0}\right)-G\left(t_{0}, \mathfrak{X}_{0}\right)\right\|}{m\left(G_{t}\right)} .
\end{aligned}
$$

5. Perturbed equations. Let $\Phi$ map $X$ into $Y$. We now consider the equation

$$
\Phi \xi=\eta
$$

Suppose $\Phi$ is unavailable in a form suitable for computations, while $F$, which is considered more suitable for computations, is an approximation for $\Phi$. If also the exact value of $\eta$ is not available, but $y$ is a convenient approximation for $\eta$, then we propose to use the solution $x$ of equation (1) as an approximation for the solution, $\xi$, of equation (3). If we let $\phi=F-\Phi, \beta=y-\eta, \alpha=x-\xi$ we see that we shall solve the "perturbed" equation

$$
(\Phi+\phi)(\xi+\alpha)=\eta+\beta .
$$

Since it may be useful to know a bound for the error $\alpha$ before the computation of $x$ is made, it is desirable to present a bound which is independent of $x$.

CRITERION 1. Let $F$ be given and let $y$ be a given element of $Y$. Assume that $M(\phi)=\delta<f$. If there exists an $x$ satisfying equation (1), then this solution is unique and

$$
\|\alpha\| \leqq \frac{\|\beta\|+\|\phi x\|}{f-\delta} \leqq \frac{f(\|\beta\|+\|\phi(\theta)\|)+\delta\|y-F(\theta)\|}{f(f-\delta)} .
$$

Proof. The condition $f>0$ implies the uniqueness. Also $f\|\alpha\|$ $\leqq\|F x-F \xi\|=\|\beta-\phi \xi\| \leqq\|\beta\|+\delta\|\alpha\|+\|\phi x\|$.

6. Output errors. We might, as an alternative to the viewpoint of $\S 5$, regard equation (3) as defining $\eta$ in terms of $\Phi$ and $\xi$. Suppose, due to previous errors in the computations, we have available, instead 
of $\Phi$ and $\xi$, approximations to them, $F$ and $x$. Then we propose to use $y=F x$ as an approximation for $\eta$. As above let $\phi=F-\Phi, \alpha=x-\xi$, $\beta=y-\eta$.

Criterion 2. Let $F$ and $x$ be given. If $M(\phi)=\delta, M(F)=C$, then $\|\beta\| \leqq(C+\delta)\|\alpha\|+\|\phi x\| \leqq(C+\delta)\|\alpha\|+\|\phi(\theta)\|+\delta\|x\|$.

PRoOF. $\beta=F x-\Phi \xi=(F x-F \xi)+(\phi \xi-\phi x)+\phi x$.

7. Characteristic value problem. Let $W$ be a set of elements satisfying the following conditions: $\left(\mathrm{h}_{1}\right) W$ is a metric space with metric function $\rho ;\left(\mathrm{h}_{2}\right)$ for each real (or complex) number $\gamma$ and each w in $W$, $\gamma w$ is in $W ;\left(h_{3}\right) W$ has a center of homogeneity, $\phi$, i.e. for each number $\gamma$ and each $w$ in $W, \rho(\gamma w, \phi)=|\gamma| \rho(w, \phi) ;\left(h_{4}\right)$ if $\left\{\gamma_{n}\right\}$ approaches $\gamma$ and $\left\{w_{n}\right\}$ approaches $w$ and $\left\{\gamma_{n} w_{n}\right\}$ approaches $w^{\prime}$, then $w^{\prime}=\gamma w$. Denote $\rho(w, \phi)$ by $L(w)$. We shall write $\gamma^{-1} w$ as $(1 / \gamma) w$ or $w / \gamma$.

Let $p$ be a positive number and let $S_{p}$ denote the set of all $w$ in $W$ such that $L(w)=p$. Let $H$ map $S_{p}$ into $W$. If there is a $\psi$ in $S_{p}$ and a number $\lambda \neq 0$ such that $(1 / \lambda) H \psi=\psi$, then we say that $\lambda$ is a $(p)$-characteristic value having the $(p)$-characteristic vector $\psi$. We assume that, for each $w$ in $S_{p}, H w \neq \phi$ and that $H$ is continuous on $S_{p}$.

Take any $\psi_{0}$ in $S_{p}$. Let $\psi_{n+1}=\left(1 / \lambda_{n}\right) H \psi_{n}$, where $\lambda_{n}=L\left(H \psi_{n}\right) / p$ $(n=0,1,2, \cdots)$. If $\left\{\psi_{n}\right\}$ converges, say to $\psi$, then, clearly, $\left\{H \psi_{n}\right\}$ converges to $H \psi,\left\{\lambda_{n}\right\}$ converges, say to $\lambda$. Then $\lambda$ is a $(p)$-characteristic value having the $(p)$-characteristic vector $\psi$. Here $\lambda>0$ but if one uses instead $\lambda_{n}=-L\left(H \psi_{n}\right) / p$, then $\lambda<0$. (In the complex case we may take $\lambda_{n}=e^{i \omega} L\left(H \psi_{n}\right) / p$.) We postpone to a later paper a study of the convergence of $\left\{\psi_{n}\right\}$, limiting ourselves here to one situation, for which the convergence will be demonstrated. We shall not require that $(\alpha \beta) w=\alpha(\beta w)$.

We shall now replace condition $\left(\mathrm{h}_{4}\right)$ by: $\left(\mathrm{h}_{5}\right)$ if $\alpha, \beta$, and $\gamma$ are numbers and $u$ and $v$ are in $W$, then $\rho(\gamma u, \gamma v)=|\gamma| \rho(u, v)$ and $\rho(\alpha u$, $\beta u)=|\alpha-\beta| L(u)$. From this it follows that if $\left\{\gamma_{n}\right\}$ converges to $\gamma$ and $\left\{w_{n}\right\}$ converges to $w$, then $\left\{\gamma_{n} w_{n}\right\}$ converges to $\gamma w$, since $\rho\left(\gamma w, \gamma_{n} w_{n}\right) \leqq \rho\left(\gamma w, \gamma w_{n}\right)+\rho\left(\gamma w_{n}, \gamma_{n} w_{n}\right) \leqq|\gamma| \rho\left(w, w_{n}\right)+\left|\gamma-\gamma_{n}\right|$ $\cdot\left(L(w)+\rho\left(w_{n}, w\right)\right)$.

THEOREM 3. Let $W$ satisfy $\left(\mathrm{h}_{1}\right),\left(\mathrm{h}_{2}\right)$, and $\left(\mathrm{h}_{5}\right)$ over the complex numbers. Let $H$ map $S_{p}$ into $W$. We assume that there is a number $M>0$ such that, for each $u, v$ in $S_{p}, \rho(H u, H v) \leqq M \rho(u, v)$. Let $W$ be complete for $H$ (for each Cauchy sequence $\left\{v_{n}\right\}$ in $S_{p},\left\{H v_{n}\right\}$ has a limit in $W$ ). Let $\inf _{v \in s_{p}} L(H v)=c$. We assume that $q=2 M p / c<1$. Let $\omega$ be any real number. Then the sequences $\left\{\psi_{n}\right\},\left\{\lambda_{n}\right\}$ defined by $\psi_{n+1}=H \psi_{n} / \lambda_{n}$, $\lambda_{n}=e^{i \omega} L\left(H \psi_{n}\right) / p(n=0,1,2, \cdots), \psi_{0}$ an arbitrary element of $S_{p}$, converge respectively to $\psi(a(p)$-characteristic vector) and $\lambda$ (the $(p)$-char- 
acteristic value for $\psi) ; \lambda$ has argument $\omega$. We have the estimates of error

$$
\begin{aligned}
\rho\left(\psi, \psi_{n}\right) & \leqq \frac{q}{1-q} \rho\left(\psi_{n}, \psi_{n-1}\right) \leqq \frac{q^{n}}{1-q} \rho\left(\psi_{1}, \psi_{0}\right), \\
\left|\lambda-\lambda_{n}\right| & =\frac{1}{p}\left|L(H \psi)-L\left(H \psi_{n}\right)\right| \leqq \frac{1}{p} \rho\left(H \psi, H \psi_{n}\right) \leqq \frac{M}{p} \rho\left(\psi, \psi_{n}\right) .
\end{aligned}
$$

Proof. We have

$$
\begin{aligned}
\rho\left(\psi_{n+1}, \psi_{n}\right) & =\rho\left(\frac{H \psi_{n}}{\lambda_{n}}, \frac{H \psi_{n-1}}{\lambda_{n-1}}\right) \\
& \leqq \rho\left(\frac{H \psi_{n}}{\lambda_{n}}, \frac{H \psi_{n-1}}{\lambda_{n}}\right)+\left|\frac{1}{\lambda_{n}}-\frac{1}{\lambda_{n-1}}\right| L\left(H \psi_{n-1}\right) \\
& =\frac{1}{\left|\lambda_{n}\right|}\left(\rho\left(H \psi_{n}, H \psi_{n-1}\right)+\left|L\left(H \psi_{n-1}\right)-L\left(H \psi_{n}\right)\right|\right) \\
& \leqq \frac{2}{\left|\lambda_{n}\right|} \rho\left(H \psi_{n}, H \psi_{n-1}\right) \leqq q \rho\left(\psi_{n}, \psi_{n-1}\right),
\end{aligned}
$$

since $\left|\lambda_{n}\right| \geqq c / p$. Thus (as in the proof of Theorem 1) $\rho\left(\psi_{n+k}, \psi_{n}\right)$ $\leqq\left(q^{n} /(1-q)\right) \rho\left(\psi_{1}, \psi_{0}\right)$, and $\left\{\psi_{n}\right\}$ is a Cauchy sequence in $S_{p}$. Since $W$ is complete for $H,\left\{H \psi_{n}\right\}$ converges, says to $w$. Then $\left\{\lambda_{n}\right\}$ converges to $e^{i \omega} L(w) / p=\lambda$. Hence $\left\{\psi_{n}\right\}$ also converges.

REMARK 6. Under the conditions of the theorem we can show the nondegeneracy, continuity, and boundedness of quantities which in the associative case are the eigenvalues.

(a) If $H \psi=\lambda \psi$ and $H v=\lambda v$ (or if $H \psi / \lambda=\psi$ and $H v / \lambda=v$ ), then $\psi=v$.

Proof. $M \rho(\psi, v) \geqq \rho(H \psi, H v)=|\lambda| \cdot \rho(\psi, v) \geqq 2 M \rho(\psi, v)$.

(b) If $H \psi=\lambda \psi$ and $H u=\mu u$, then

$$
\frac{|\lambda|+M}{p} \rho(\psi, u) \geqq|\lambda-\mu| \geqq \frac{|\lambda|-M}{p} \rho(\psi, u) .
$$

ProOF.

$$
\begin{aligned}
M \rho(\psi, u) & \geqq|\rho(H \psi, \lambda u)-\rho(\lambda u, H u)| \\
& =|| \lambda|\cdot \rho(\psi, u)-| \lambda-\mu|\cdot L(u)| .
\end{aligned}
$$

(c) If $H \psi=\lambda \psi$ and $H u=\mu u$, then $0<|\lambda|-2 M \leqq|\mu| \leqq|\lambda|+2 M$.

Proof. $2 p M \geqq M \rho(\psi, u) \geqq \rho(H \psi, H u) \geqq p|| \lambda|-| \mu||$.

REMARK 7. As an illustration of a situation satisfying the conditions of the theorem let $x_{0}$ be a fixed element of a Banach space $\mathfrak{X}$ 
with $\left\|x_{0}\right\|=4$. For each $x$ in $\mathfrak{X}$ let $H x=x_{0}+x$. Then, with $p=1$ we have $M=1, c=3, q=2 / 3$.

REMARK 8. If, in addition to the hypotheses of the theorem, $H$ maps $S_{p}$ onto a subset of $S_{k}$, then $c=k$ and $\left|\lambda_{n}\right|=k / p$. From the inequality $\rho\left(\psi_{n+1}, \psi_{n}\right)=(p / k) \rho\left(H \psi_{n}, H \psi_{n-1}\right) \leqq(p M / k) \rho\left(\psi_{n}, \psi_{n-1}\right)$, we see that we can replace the condition $q=2 M p / c<1$ of Theorem 3 by the condition $q^{\prime}=M p / k<1$ and, in the estimates of error, the $q$ may be replaced by $q^{\prime}$.

The writer wishes to thank Professors H. Goheen, C. Langenhop, H. P. Thielman, and B. Vinograde for many helpful discussions concerning the material presented here.

Iowa State College of Agriculture and Mechanic Arts 